\title{
Association between investigator-measured body-mass index and colorectal adenoma: a systematic review and meta-analysis of 168,201 subjects
}

\author{
Martin Chi-sang Wong ${ }^{1,2} \cdot$ Chun-hei Chan ${ }^{2} \cdot$ Wilson Cheung ${ }^{2} \cdot$ Din-hei Fung ${ }^{2} \cdot$ Miaoyin Liang $^{2}$. \\ Jason Li-wen Huang ${ }^{2} \cdot$ Yan-hong Wang $^{3} \cdot$ Johnny Yu Jiang ${ }^{4} \cdot$ Chun-pong $\mathrm{Yu}^{5} \cdot$ Harry Haoxiang Wang ${ }^{6}$. \\ Justin Che-yuen $\mathrm{Wu}^{1,7,8} \cdot$ Francis Ka-leung Chan ${ }^{1,7,8} \cdot$ Joseph Jao-yiu Sung ${ }^{1,7,8}$
}

Received: 26 June 2017 / Accepted: 23 November 2017 / Published online: 29 December 2017

(C) The Author(s) 2017. This article is an open access publication

\begin{abstract}
The objective of this meta-analysis is to evaluate the odds of colorectal adenoma (CRA) in colorectal cancer screening participants with different body mass index (BMI) levels, and examine if this association was different according to gender and ethnicity. The EMBASE and MEDLINE were searched to enroll high quality observational studies that examined the association between investigator-measured BMI and colonoscopy-diagnosed CRA. Data were independently extracted by two reviewers. A random-effects meta-analysis was conducted to estimate the summary odds ratio (SOR) for the association between BMI and CRA. The Cochran's Q statistic and $\mathrm{I}^{2}$ analyses were used to assess the heterogeneity. A total of 17 studies (168,201 subjects) were included. When compared with subjects having BMI $<25$, individuals with BMI 25-30 had significantly higher risk of CRA (SOR $1.44,95 \%$ CI $1.30-1.61 ; \mathrm{I}^{2}=43.0 \%$ ). Subjects with BMI $\geq 30$ had similarly higher risk of CRA (SOR $1.42,95 \%$ CI $1.24-1.63 ; \mathrm{I}^{2}=18.5 \%$ ). The heterogeneity was mild to moderate among studies. The associations were significantly higher than estimates by previous meta-analyses. There was no publication bias detected (Egger's regression test, $p=0.584$ ). Subgroup analysis showed that the magnitude of association was significantly higher in female than male subjects (SOR 1.43, 95\% CI 1.30-1.58 vs. SOR 1.16, 95\% CI 1.07-1.24; different among different ethnic groups (SOR 1.72, 1.44 and 0.88 in White, Asians and Africans, respectively) being insignificant in Africans; and no difference exists among different study designs. In summary, the risk conferred by BMI for CRA was significantly higher than that reported previously. These findings bear implications in CRA risk estimation.
\end{abstract}

Keywords Body mass index $\cdot$ Colorectal adenoma $\cdot$ Association $\cdot$ Meta-analysis

$\begin{array}{ll}\text { Abbreviations } \\ \text { BMI } & \text { Body-mass index } \\ \text { CRA } & \text { Colorectal adenoma } \\ \text { NOS } & \text { Newcastle Ottawa Scale (NOS) } \\ \text { SOR } & \text { Summary odds ratio } \\ \text { SAT } & \text { Subcutaneous adipose tissue } \\ \text { VAT } & \text { Visceral adipose tissue }\end{array}$

Electronic supplementary material The online version of this article (https://doi.org/10.1007/s10654-017-0336-x) contains supplementary material, which is available to authorized users.

Joseph Jao-yiu Sung

jjysung@cuhk.edu.hk

Extended author information available on the last page of the article

\section{Introduction}

Colorectal cancer (CRC) is a leading cause of morbidity and mortality on a global scale [1]. Its incidence is rising rapidly in many low- and middle-income countries [2], as well as Asia Pacific nations such as Japan, Korea, Singapore and Hong Kong [3, 4]. Overweight and obesity, defined as a body mass index (BMI) of $25-30$ and $\geq 30 \mathrm{~kg} /$ $\mathrm{m}^{2}$, respectively, is one of the recognized environmental risk factors for the development of CRC [5-7]. Whilst obesity is preventable, statistics from the World Health Organization reported that more than 1.9 billion adults aged 18 years or older (39\%) were overweight in 2014; amongst them over 600 million (13\%) were obese [8]. Its increasing prevalence has been regarded as a major contributor to the rising trend of CRC. 
Colorectal adenomas (CRA) are present in more than $30 \%$ of asymptomatic general populations [9]. Among all CRC screening participants who received colonoscopy with polyps detected, CRA is amongst the most frequent pathological findings [10]. Since most CRCs develop via genetic and morphological adenoma-carcinoma progression from CRAs, it is widely accepted that both CRCs and CRAs share similar epidemiological features and etiological causes. Hence, some risk algorithms have adopted BMI as a predictor variable to risk-stratify subjects for colorectal neoplasia [10].

Nevertheless, the association between BMI and CRA has not been consistently demonstrated in all populations [11-28]. Some studies reported a significant association between BMI and CRA [11, 12, 14-21, 23-26, 28] whilst others did not [13, 22, 27, 29-31]. Two recent meta-analyses have been performed to pool data from published studies on the relationship between BMI and CRA. In 2012, Okabayashi and colleagues systematically reviewed 23 studies (105,190 participants) in their meta-analysis on the prediction value of BMI for CRA, and revealed a doseresponse relationship where the risk of CRA increased with higher BMI levels [32]. However, there exist major limitations as self-reported BMI was used, and this could lead to misclassification of BMI categories in public health research [33]. In that meta-analysis [32], a significant proportion of studies included used self-reported questionnaires to determine BMI and the presence of CRA, and this could reduce the robustness of the conclusions drawn due to possible reporting bias. In another systematic review with the same research objective, the limitation of relying on questionnaire surveys to measure BMI or CRA was noticed in 15 of 36 included studies [34]. Since the publication of these two meta-analyses, there are 10 additional studies that were published including large number of screening participants using physician-measured BMI and colonoscopy diagnosed CRA as inclusion criteria. For instance, a multi-centre study in 16 Asia Pacific countries recruited more than 11,797 asymptomatic screening participants who received colonoscopies, and the study was published in 2016 [35]. The precise magnitude of the association between BMI and CRA remains unknown, and whether there exist differences in this association in subjects with different characteristics is yet to be explored. This knowledge gap is important as it bears clinical implications in formulation of risk scores for CRA in different patient groups, and informs clinical guidelines regarding target groups for priority screening. This metaanalysis aims to evaluate the odds of colorectal adenoma (CRA) in colorectal cancer screening participants with different body mass index (BMI) levels, and examine if this association was different according to gender and ethnicity.

\section{Methods}

\section{Literature search strategy}

We conducted the literature search by systematically searching MEDLINE (from 1946 to March 2017), EMBASE (from 1974 to March 2017) and by hand searching the reference lists of original studies and review articles on this topic. Our search terms consisted of three main components, colorectal (colorectal OR colon OR colonic OR rectum OR rectal) AND disease (cancer* OR neoplas* OR tumor* OR tumour* OR carcinoma* OR sarcoma* OR adenoma* OR lesion* OR polyp* OR CRC) AND obesity or overweight (body mass index OR BMI OR body size OR body weight OR intraabdominal OR overweight OR fat OR obesity OR obese OR waist) [32] (Supplementary File 1). Grey literature search was performed in Grey Literature Report (www.greylit.org), related thesis, and conference reports. No language restrictions were imposed.

\section{Inclusion and exclusion criteria}

CRA, defined as the presence of either non-advanced or advanced adenoma, is the primary outcome of this study. We included all cross-sectional studies, case control studies and cohort studies that examined the relationship between BMI and the prevalence of CRA. In these studies, odds ratios (OR) with $95 \%$ confidence intervals (CI) between CRA and BMI categories were recorded. We excluded the following studies: (1). those with hyperplastic polyps, serrated adenomas or CRC cases as majority of all lesions; (2). those where subjects had higher CRC risk as compared to the general population, for instance, individuals with a family history of CRC in first-degree relatives; (3). those that could not generate OR for BMI category and CRAs; (4). those with symptomatic participants; (5). those with BMI data obtained from self-reported questionnaires; (6). those with CRA not diagnosed by colonoscopy and histological examination; (7). those with CRA data not derived from the whole colon and rectum. The eligibility of studies was assessed by two investigators (J. L. H. and C. H. C.) and in cases of disagreement, consensus was made via referral to a third reviewer (M. C. S. W.). We attempted to contact authors of studies if there were any missing data.

\section{Quality assessment of selected studies}

The Newcastle Ottawa Scale (NOS) was employed to evaluate the quality of the included studies according to their design by two assessors (J. L. H. and C. H. C.) who are librarian experts [36, 37]. The NOS was used to 
confirm that the included studies are of high quality, which was scored based on the summation of items described below. Similar items among different study types for quality assessment were as follows: (1) representativeness of the samples: one point was assigned if the subjects represent the general population/case group/controls group/exposed cohort/non-exposed cohort. No points were assigned if samples are special population groups (e.g. veteran) or not mentioned; (2) ascertainment of the exposure: one point was assigned if measurement of BMI was performed by healthcare professionals, 0 point was assigned if BMI was self-reported or not specified. Since all our included studies measured BMI by healthcare professionals, none was assigned 0 point; (3) comparability: for subjects in different outcome groups or case/control groups, two points were assigned for adequate adjustment of recognized risk factors for colorectal adenoma; one point for adjustment of some covariates only, and zero point for no adjustment; (4) assessment of the outcome: colonoscopy and histological examination: one point was assigned if it was based on medical records or histology report, no point was given if the assessment was self-reported by study participants or not specified.

For cross-sectional studies, additional items for quality assessment include: (1) sample size: if the sample size is justified, one point was assigned, otherwise no point was given; (2) non-respondents: one point was assigned if the response rate is satisfactory, otherwise no point; (3) assessment of the outcome: for those studies in which outcome assessment was independent and blinded, one extra point was added accordingly; (4) statistical test: one point was assigned if the statistical test is appropriate, clearly described and complete; otherwise no point was assigned. For case-control studies, additional items include: (1) same method of ascertainment for cases and controls: one point was assigned; (2) non-response rate: one point was given if the rate for both case and control groups was the same, and no point was assigned for nonrespondents. For cohort studies, the additional items are: (1) demonstration that outcome of interest was not present at study commencement: one point was assigned for stating exclusion of CRA/ advanced CRA/ CRC subjects or stating subjects have no history of CRA/ advanced CRA/ CRC; (2) follow-up duration: one point was assigned for all eligible studies if the follow-up period is long enough to detect CRA; (3) adequacy of following up of cohorts: one point was assigned for completing at least $90 \%$ of follow-up. Scores ranged from 0 (lowest) to 9 (highest). Similar to previous literature [32], studies with scores $\geq 7$ were classified as "high" quality and those with scores $<7$ were classified as "low" quality.

\section{Data extraction}

The characteristics of studies were recorded, including the names of the first authors, publication year, country, design, enrolment year, BMI category, strategies to capture BMI data, the definition of non-cases and the definition of advanced adenoma. BMI is categorized according to WHO classification: normal $\left(<25 \mathrm{~kg} / \mathrm{m}^{2}\right)$, overweight $\left(25-30 \mathrm{~kg} / \mathrm{m}^{2}\right)$ and obese $\left(\geq 30 \mathrm{~kg} / \mathrm{m}^{2}\right)$. The number of cases and non-cases in the 3 categories, as well as the study design, gender and subject ethnicity, were recorded if available. Data extraction and data checking were performed by 3 investigators (J. L. H., C. H. C. and W. C.)

\section{Statistical analysis}

Random effects model meta-analysis was conducted to synthesize a summary estimate of the association between different BMI groups and CRA. Summary odds ratios (SOR) with $95 \%$ confidence intervals (CI) were used as a proxy measure for effect size, and were calculated by comparing 3 BMI categories $\left(\geq 25,25-30, \geq 30 \mathrm{~kg} / \mathrm{m}^{2}\right)$ with BMI $<25 \mathrm{~kg} / \mathrm{m}^{2}$. The SOR was computed with the assumption that the outcomes categorized by different BMI groups were derived from patients independently, so there was no within-study correlation of adenoma prevalence. Z-tests were used to investigate the significance of pooled estimate, and Cochran's Q and $\mathrm{I}^{2}$ statistics were used to examine the heterogeneity within groups and between groups [38]. For publication bias, funnel plot asymmetry was assessed by the Egger's and Begg's regression test [39, 40]. Subgroup analysis was applied in this study to perform comparisons according to subsets of studies, such as study design, gender, ethnicity, types of adenoma and degree of CRA progression. We also conducted a metaregression analysis to explore heterogeneity between the studies.

In the present study, R ver. 3.3.1 (The R Foundation for Statistical Computing) with metafor package ver. 1.9-9 was used to conduct the statistical analysis [41]. All functions were performed under restricted maximum likelihood estimation. Two-tailed $p$ value $<0.05$ was defined as statistical significant for all the comparisons. Heterogeneity was considered as low, moderate and high, when $\mathrm{I}^{2}$ was 25,50 and $75 \%$ respectively. This systematic review was written following the PRISMA guideline [42]. 


\section{Results}

\section{Search results and study characteristics}

The search strategy yielded 3292 citations. We removed 1027 duplicates, and 2173 articles were removed after title and abstract review (Fig. 1). A total of 92 studies were reviewed in full text and 13 studies fulfilled our eligibility criteria. Four additional articles were retrieved from review of the reference sections of original articles and grey literature search, resulting in 17 articles included for data analysis (168,201 subjects). Among them, 12 were crosssectional studies [24, 34, 43-52], 4 were case-control studies [53-56], and one was a cohort study [57] (Tables 1, 2 ). The quality of all included studies was assessed by the Newcastle Ottawa Scale (NOS) (Table 3). All studies were found to have good quality, with 15 studies scoring 8 points and 2 studies scoring 7 points. Of all the 17 studies, 11 included data of Asian subjects [24, 44-51, 53, 56], 4 included data of white subjects and 4 included data of individuals of African descent [46, 52, 55, 56]. The proportion of screening participants with $\mathrm{BMI}>25 \mathrm{~kg} / \mathrm{m}^{2}$ was 29.3, 49.7 and $58.1 \%$ in Asian, African and white subjects, respectively. No studies were found to use identical cohorts. The search did not identify any studies published in grey literature.

\section{The association between body mass index and colorectal adenoma}

Meta-analysis of the included articles via a random-effects model showed a SOR of 1.42 (95\% CI 1.34, 1.51) among subjects with $\mathrm{BMI} \geq 25$ compared to subjects with $\mathrm{BMI}$ $<25$, where the heterogeneity was moderate and statistically insignificant $\left(\mathrm{I}^{2}=34.3 \%, \quad\right.$ pheterogeneity $\left.^{2}=0.063\right)$ (Fig. 2a). Using BMI $<25$ as a reference, the associations with any CRA were similar between those with BMI 25-30 (SOR 1.44, 95\% CI 1.30, 1.61; $\mathrm{I}^{2}=43.0 \%$, pheterogeneity $=0.099$; Fig. 2b) and BMI $\geq 30$ (SOR 1.42, 95\% CI $1.24,1.63 ; \mathrm{I}^{2}=18.5 \%$, pheterogeneity $=0.193$; Fig. $2 \mathrm{c}$ ). No statistically significant difference were found between the two groups [ $p$ difference $=0.887$ ]. All 17 studies reported data on CRA among subjects with BMI $>25$, but only 10 studies reported number of CRA among subjects with BMI
Fig. 1 Flow diagram of study selection
Potential articles identified through literature search from Embase and Medline $(n=3,292)$

Duplicates removed $(\mathrm{n}=1,027)$
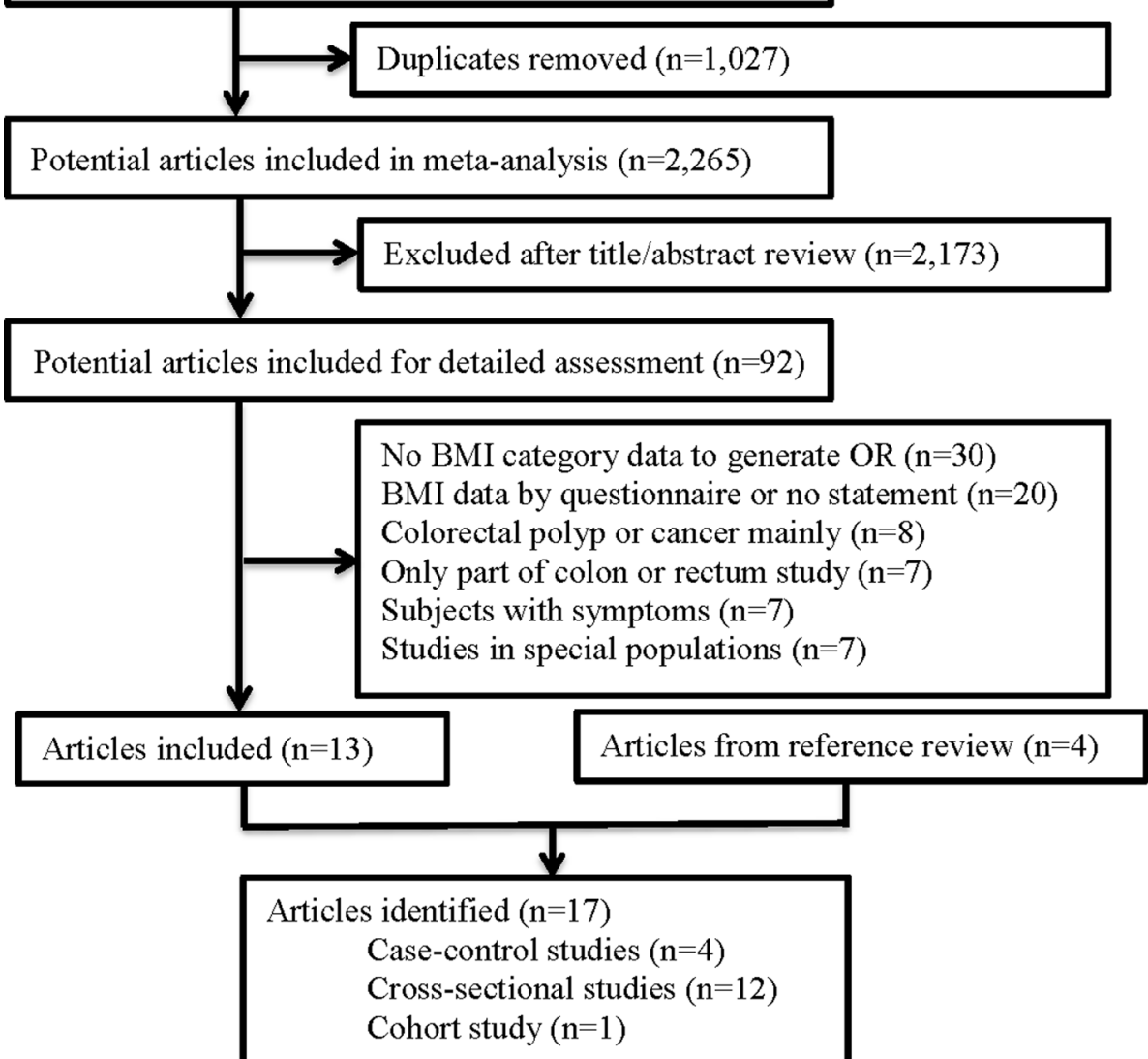
Table 1 Characteristics of included studies

\begin{tabular}{|c|c|c|c|c|c|c|c|}
\hline Authors & Year & Country & Design & Sample size & BMI category & $\begin{array}{l}\text { Prevalence }(\%) \text { of subjects } \\
\text { with BMI }>25 \mathrm{~kg} / \mathrm{m}^{2}\end{array}$ & $\begin{array}{l}\text { NOS } \\
\text { score }\end{array}$ \\
\hline Guilera et al. [43] & 2005 & USA & 1 & 720 & $18.5-24.9,25-29.9,>30$ & 61.9 & 8 \\
\hline Kim et al. [44] & 2007 & South Korea & 1 & 1744 & $18.5-23.0,23.0-24.9, \geq 25$ & 31.1 & 8 \\
\hline Sedjo et al [57] & 2007 & USA & 3 & 600 & $<25,25-29,30+$ & 82.8 & 8 \\
\hline Kim et al. [45] & 2010 & South Korea & 1 & 1316 & $<25, \geq 25$ & 30.9 & 8 \\
\hline Nam et al. [24] & 2010 & South Korea & 1 & 3933 & $<20,20-24.9,25.0-29.9, \geq 30$ & 33.4 & 8 \\
\hline Stein et al. [46] & 2010 & USA & 1 & 600 & $<25,25-30,30-35, \geq 35$ & 68.3 & 8 \\
\hline Kim et al. [47] & 2011 & South Korea & 1 & 1322 & $<23,23.0-24.9, \geq 25$ & 37.4 & 8 \\
\hline Kim et al. [48] & 2012 & South Korea & 1 & 3430 & $18.5-25, \geq 25$ & 29.9 & 8 \\
\hline Choe et al. [53] & 2013 & South Korea & 2 & 1206 & $\leq 22.9,23.0-24.9,25.0-29.9, \geq 30$ & 34.4 & 8 \\
\hline Czwornog et al. [54] & 2013 & USA & 2 & 773 & $18.5-25,25.0-30, \geq 30$ & 72.7 & 8 \\
\hline Lipka et al. [55] & 2013 & USA & 2 & 779 & $<18.5,18.5-24.9,25.0-29.9,>30.0$ & 82.4 & 7 \\
\hline Yun et al. [49] & 2013 & South Korea & 1 & 18,085 & $<18.5,18.5-22.9,23.0-24.9, \geq 25$ & 18.4 & 8 \\
\hline Lee et al. [50] & 2014 & South Korea & 1 & 1574 & $<23,23-25, \geq 25$ & 32.0 & 8 \\
\hline Wang et al. [51] & 2014 & Taiwan & 1 & 1894 & $<25,25-30,>30$ & 34.1 & 8 \\
\hline Murphy et al. [52] & 2015 & South Korea & 2 & 3561 & $<25, \geq 25$ & 60.2 & 8 \\
\hline Kim et al. [56] & 2015 & USA & 1 & 2184 & $18.5-25,25-30,>30$ & 33.4 & 7 \\
\hline Wong et al. [35] & 2016 & Asia Pacific & 1 & 11,362 & $<25,25-30, \geq 30$ & 32.2 & 8 \\
\hline
\end{tabular}

Design: 1 cross-sectional, 2 case-control, 3 Cohort

$B M I$ body mass index, NOS scale the Newcastle-Ottawa scale

Table 2 Pathology findings from included studies

\begin{tabular}{lclcllll}
\hline Author & Polyp-free (n, \%) & HP (n, \%) & Non-AA (n, \%) & AA (n, \%) & CRC (n, \%) & Definition of normal & Definition of AN \\
\hline Guilera et al. [43] & $494(68.6)^{\#}$ & & $226(31.4)$ & NS & NS & 1 & NS \\
Kim et al. [44] & $1460(83.7)$ & NS & $206(11.8)$ & $78(4.5)$ & NS & 2 & AA, CRC \\
Sedjo et al. [57] & $410(68.3)$ & $54(9.0)$ & $98(16.3)$ & $38(6.3)$ & 0 & 1 & AA \\
Kim et al. [45] & $1053(80.0)$ & Excluded & $228(17.3)$ & $35(2.7)$ & Excluded & 2 & AA \\
Nam et al. [24] & $2877(73.2)$ & NS & $960(24.4)$ & $85(2.2)$ & $11(0.3)$ & 2 & AA, CRC \\
Stein et al. [46] & $384(64.0)$ & NS & $176(29.3)$ & $40(6.7)$ & 0 & 2 & AA, CRC \\
Kim et al. [47] & $908(68.7)$ & Excluded & $368(27.8)$ & $46(3.5)$ & Excluded & 2 & AA \\
Kim et al. [48] & $2456(71.6)^{\#}$ & & $744(21.7)$ & $224(6.5)$ & $6(0.2)$ & 2 & AA, CRC \\
Choe et al. [53] & $557(46.2)$ & NS & $554(45.9)$ & NS & $153(12.7)$ & 3 & NS \\
Czwornog et al. [54] & $567(73.4)$ & NS & $206(26.6)$ & NS & NS & 1 & AA (any size) \\
Lipka et al. [55] & $612(78.6)$ & NS & $167(21.4)$ & NS & Excluded & 1 & NS \\
Yun et al. [49] & $16,163(89.4)^{\#}$ & & $1674(9.3)$ & $248(1.4)$ & Excluded & 3 & AA, CRA $\geq 3$ \\
Lee et al. [50] & $1080(68.6)^{\#}$ & & $494(31.4)$ & NS & Excluded & 1 & NS \\
Wang et al. [51] & $1379(72.8)$ & $210(11.1)$ & $305(16.1) *$ & & NS & 3 & NS \\
Murphy et al. [52] & $3129(87.9)$ & NS & $685(19.2)$ & $143(4.0)$ & $13(0.4)$ & 2 & AA, CRC \\
Kim et al. [56] & $1555(71.2)$ & NS & $629(28.8)$ & NS & NS & 1 & NS \\
Wong et al. [35] & $7177(63.2)$ & $853(7.5)$ & $2604(22.9)$ & $657(5.8)$ & $71(0.6)$ & 2 & AA \\
\hline
\end{tabular}

Normal definition: 1: non-adenomatous; 2: polyps-free; 3: normal findings

$H P$ hyperplastic polyp, $A N$ advanced neoplasia, $C R A$ colorectal adenoma, $C R C$ colorectal cancer, $A A$ advanced adenoma, adenoma measuring $>10 \mathrm{~mm}$ in diameter and/or with villous components and/or showing high grade dysplasia (32)

"Mixed with polys-free and HP (hyperplastic polyp)

*Mixed with any adenomas 


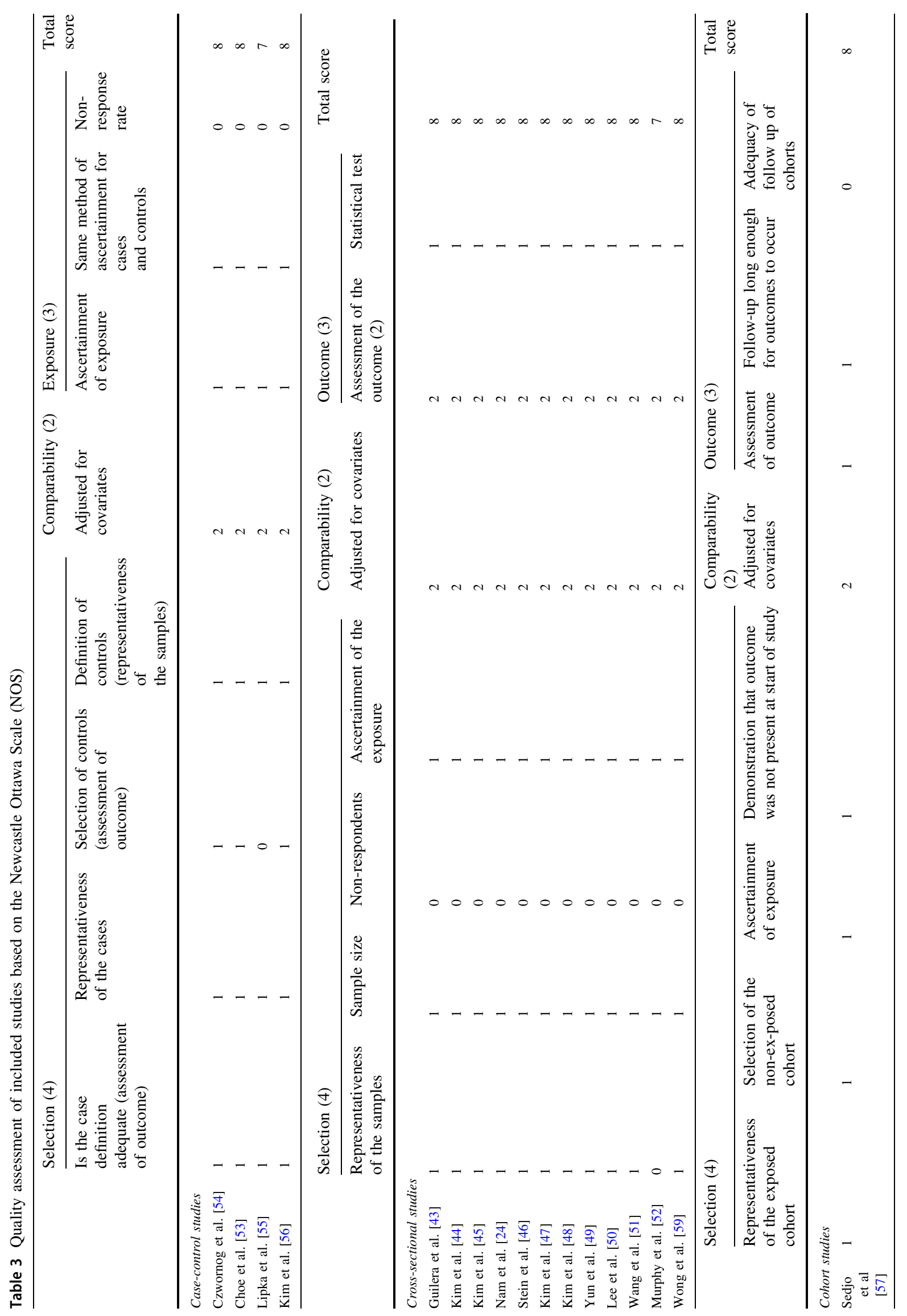


(A) BMI>25 vs $<25$

\begin{tabular}{|c|c|c|c|c|c|c|c|c|c|c|c|}
\hline \multicolumn{2}{|c|}{ A) $B M I>25$ vs $<25$} & \multicolumn{2}{|c|}{ BMI $<25$} & \multicolumn{2}{|c|}{ BMI>25 } & \multirow[b]{2}{*}{ Weights } & \multirow[b]{2}{*}{ Odds Ratio $[95 \% \mathrm{Cl}]$} & \multirow{2}{*}{\multicolumn{2}{|c|}{$\begin{array}{l}\text { Favours } \\
\text { BMI<25 }\end{array}$}} & \multirow{2}{*}{$\begin{array}{l}\text { Favours } \\
\text { BMI>25 }\end{array}$} & \\
\hline Author & Year & CRA* & CRA. & CRA- & CRA. & & & & & & \\
\hline Guilera & 2005 & 77 & 197 & 149 & 297 & $3.09 \%$ & $1.28[0.92,1.78]$ & & - & $. \quad-\quad$ & \\
\hline Kim SE & 2007 & 191 & 978 & 89 & 439 & $4.13 \%$ & $1.04[0.79,1.37]$ & & $\longmapsto$ & -1 & \\
\hline Sedjo & 2007 & 17 & 86 & 119 & 378 & $1.19 \%$ & $1.59[0.91,2.79]$ & & $\vdash$ & 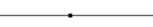 & \\
\hline Kim KS & 2010 & 173 & 737 & 90 & 316 & $3.88 \%$ & $1.21[0.91,1.62]$ & & . & $=\quad-1$ & \\
\hline Nam SY & 2010 & 631 & 1980 & 414 & 897 & $9.58 \%$ & $1.45[1.25,1.68]$ & & & $\mapsto-1$ & \\
\hline Stein & 2010 & 50 & 140 & 166 & 244 & $2.43 \%$ & $1.90[1.30,2.78]$ & & & $\longmapsto$ & \\
\hline Kim YJ & 2011 & 229 & 596 & 183 & 310 & $5.18 \%$ & $1.54[1.21,1.95]$ & & & $\longmapsto-1$ & \\
\hline Kim MC & 2012 & 647 & 1758 & 327 & 698 & $8.78 \%$ & $1.27[1.09,1.49]$ & & & $\mapsto-1$ & \\
\hline Choe & 2013 & 338 & 391 & 216 & 166 & $4.83 \%$ & $1.51[1.17,1.93]$ & & & $\longmapsto$ & \\
\hline Czwornog & 2013 & 50 & 161 & 156 & 406 & $2.56 \%$ & $1.24[0.86,1.79]$ & & $\longmapsto$ & . $\quad 1$ & \\
\hline Lipka & 2013 & 27 & 110 & 140 & 502 & $1.70 \%$ & $1.14[0.72,1.80]$ & & & -1 & \\
\hline Yun & 2013 & 1471 & 13282 & 451 & 2881 & $12.20 \%$ & $1.41[1.26,1.58]$ & & & $1-1$ & \\
\hline Lee & 2014 & 306 & 764 & 188 & 316 & $5.66 \%$ & $1.49[1.19,1.86]$ & & & $\longmapsto$ & \\
\hline Wang & 2014 & 161 & 949 & 144 & 430 & $4.75 \%$ & $1.97[1.53,2.54]$ & & & $\longmapsto \quad 1$ & \\
\hline Kim DH & 2015 & 487 & 1885 & 354 & 835 & $8.78 \%$ & $1.64[1.40,1.92]$ & & & $\mapsto-1$ & \\
\hline Murphy & 2015 & 197 & 618 & 397 & 837 & $6.62 \%$ & $1.49[1.22,1.82]$ & & & $\longmapsto$ & \\
\hline Wong MC & 2016 & 2065 & 5586 & 1196 & 2444 & $14.65 \%$ & $1.32[1.22,1.44]$ & & & 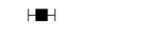 & \\
\hline \multicolumn{6}{|c|}{ Random Effect Model } & $100.00 \%$ & $1.42[1.34,1.51]$ & & & $\bullet$ & \\
\hline \multicolumn{12}{|c|}{ Overall: $Z=10.98, p<.001$} \\
\hline \multicolumn{12}{|c|}{ Heterogeneity: $\wedge^{\wedge} 2=34.26 \% ; Q(d f=16)=25.42, p=.063$} \\
\hline & & & & & & & & 0.5 & 1.0 & 2.0 & 4.0 \\
\hline & & & & & & & & & & Odds ratio & \\
\hline
\end{tabular}

(B) BMI25-30 vs $<25$

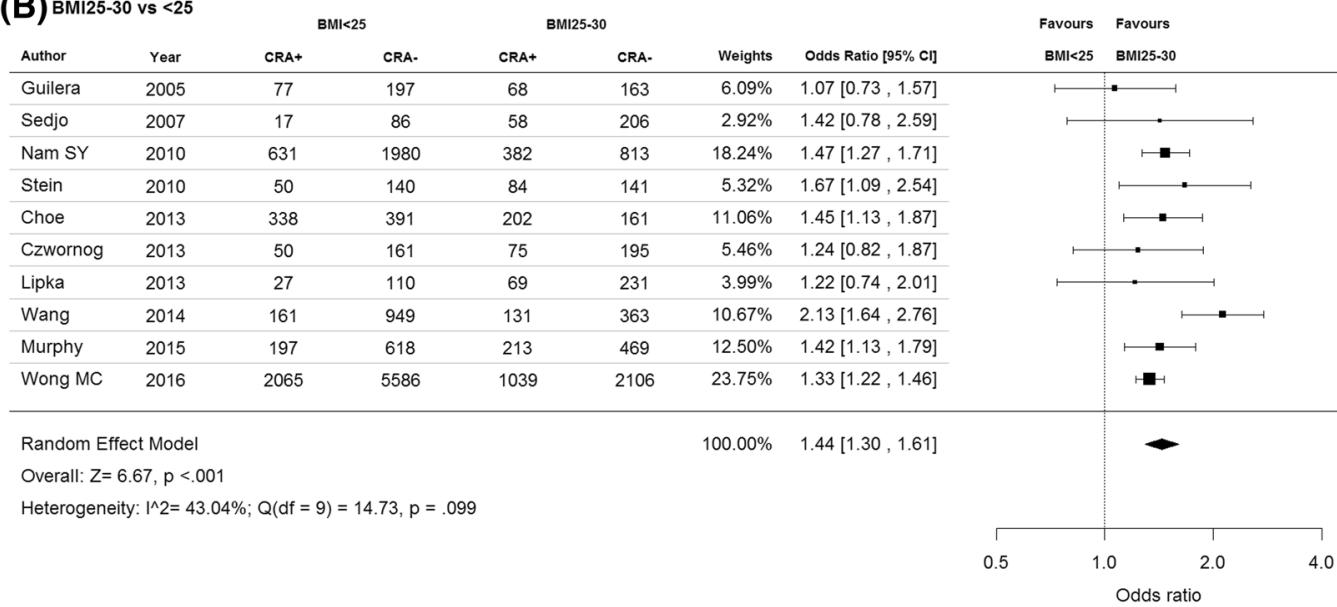

(C) $B M I>30$ vs $<25$

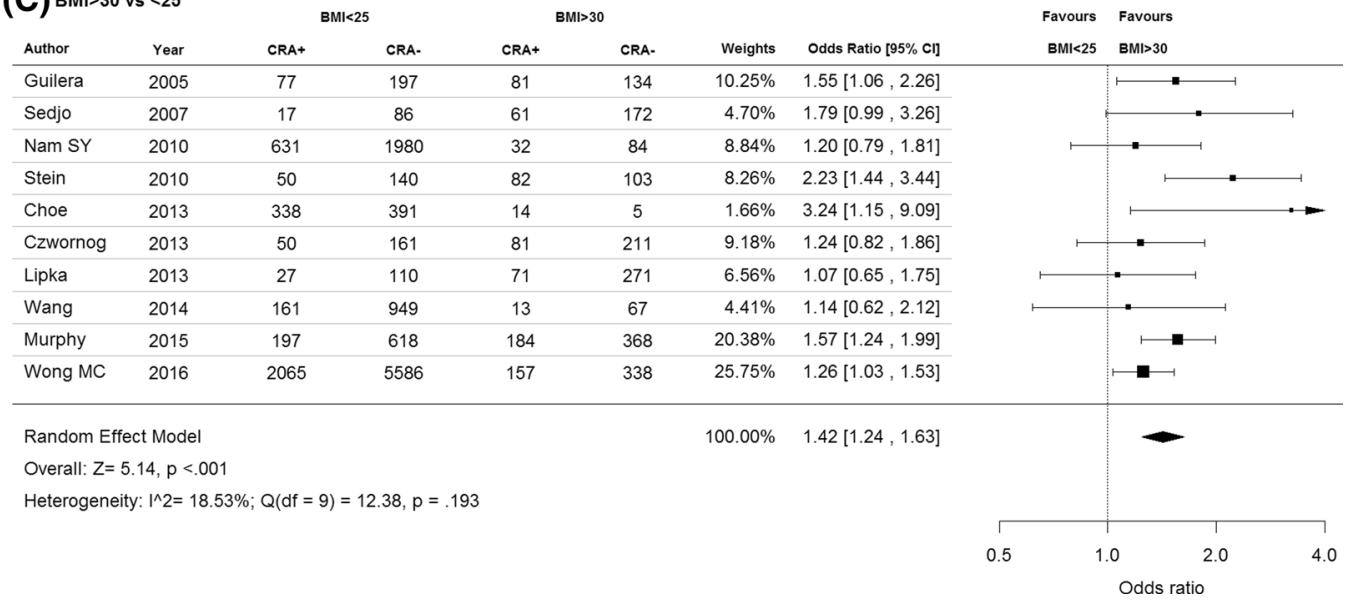

Fig. 2 Odds ratios for colorectal adenoma (a BMI $\geq 25$ vs. $<25$; b BMI $25-30$ vs. $<25$; $\mathbf{c} \geq 30$ vs. $<25$ ) 
25-30 and BMI $>30 \mathrm{~kg}^{2} / \mathrm{m}$. The magnitude of association was similar between different BMI groups and non-advanced adenoma $(\mathrm{BMI} \geq 25$ vs. $<25$ : SOR $1.36,95 \% \mathrm{CI}$ 1.26, 1.47; BMI 25-30 vs. <25: SOR $1.33,95 \%$ CI 1.22 , 1.47 ; BMI $\geq 30$ vs. $<25$ : SOR $1.38,95 \%$ CI $1.04,1.84$ ) and did not show statistically significant difference when compared with any CRA. When compared with subjects with $\mathrm{BMI}<25$, the odds of advanced adenoma was significantly higher among those with BMI $\geq 25$ (SOR 1.52 , 95\% CI 1.32, 1.73). The relationship between BMI and advanced adenoma using "non-advanced adenoma" as non-cases did not show statistical significance.

\section{Subgroup analysis}

Eight studies examined the association in men and women separately, and it was found that female subjects had significantly higher odds of CRA (SOR $1.43,95 \%$ CI 1.30, 1.58) when compared with men (SOR 1.16, 95\% CI 1.07, 1.24; between-groups $p$ difference of $<0.001$ ) (Fig. 3). Among subjects of white ethnicity (SOR 1.72, 95\% CI 1.44, 2.07) and Asian ethnicity (SOR 1.44, 95\% CI 1.32, 1.57 ), individuals with BMI $>25 \mathrm{~kg} / \mathrm{m}^{2}$ had higher odds of CRA than those with BMI $<25 \mathrm{~kg} / \mathrm{m}^{2}$. The odds was higher compared to Africans but the findings indicated only a significant difference between Asian and Africans. The SORs between BMI and CRA showed no statistically significant difference between cross-sectional and case control studies $(p=0.479)$. Meta-regression analysis based on BMI $25-30$ as a reference and $\mathrm{BMI}>30 \mathrm{~kg} / \mathrm{m}^{2}$ implied that different levels of BMI could not explain the heterogeneity observed in this meta-analysis (coefficient -0.01 [95\% CI $-0.20,0.18], p=0.905)$.

\section{Publication bias}

The Egger's test $(\mathrm{t}=-0.560, p=0.584)$ and Begg's test (Kendall's tau $=0.059, p=0.777$ ) for funnel plot asymmetry identified insignificant publication bias (Fig. 4). There were two outliers in the funnel plot, and the trim and fill analysis showed no missing studies. When these two outliers $[44,51]$ were excluded and the association between any CRA and BMI $(\geq 25$ vs. $<25)$ was re-examined, the

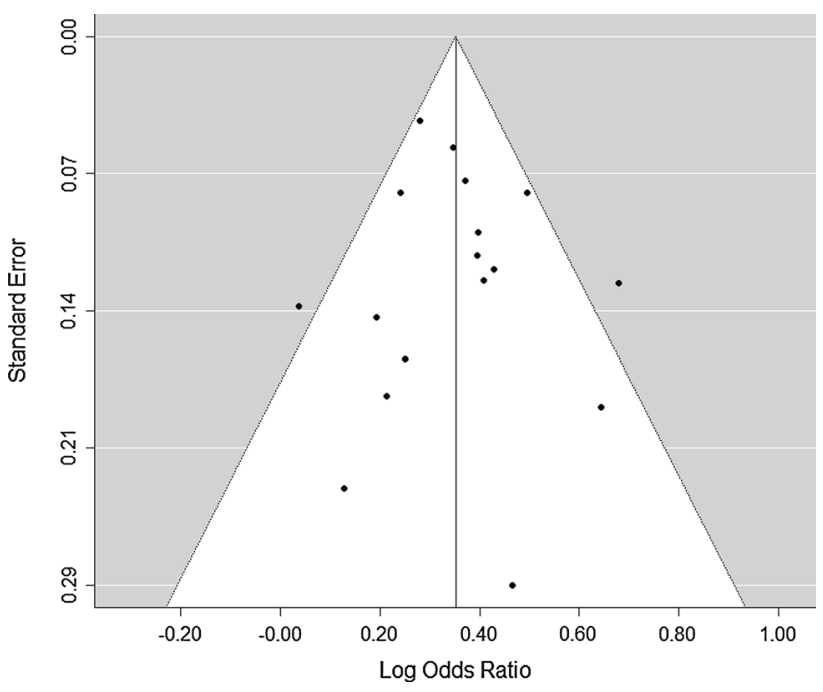

Fig. 4 Funnel Plot for identification of publication bias

\begin{tabular}{|c|c|c|c|c|c|c|c|c|}
\hline Catergory & No. of Studies & Odds Ratio [95\% Cl] & P-significance & $12(\%)$ & P-heterogeneity & P-difference & $\begin{array}{c}\text { Favours } \\
\text { BMI<25 }\end{array}$ & $\begin{array}{l}\text { Favours } \\
\text { BMI>25 }\end{array}$ \\
\hline \multicolumn{9}{|l|}{ Design } \\
\hline Case-control & 4 & $1.49[1.28,1.72]$ & $<.001$ & 19.4 & .304 & Ref & & $\mapsto-1$ \\
\hline Cross-sectional & 12 & $1.40[1.31,1.50]$ & $<.001$ & 33.7 & .067 & .479 & & 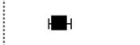 \\
\hline \multicolumn{7}{|l|}{ Gender } & & \\
\hline Male & 8 & $1.16[1.07,1.24]$ & $<.001$ & 9.4 & .225 & Ref & & $=$ \\
\hline Female & 8 & $1.43[1.30,1.58]$ & $<.001$ & 0 & .374 & $<.001$ & & +-1 \\
\hline \multicolumn{7}{|l|}{ Ethnicity } & & \\
\hline Asian & 11 & $1.44[1.32,1.57]$ & $<.001$ & 47.9 & .036 & Ref & & $H$ \\
\hline African descent & 4 & $0.88[0.61,1.29]$ & .516 & 0 & .680 & .013 & & $\longrightarrow$ \\
\hline White & 4 & $1.72[1.44,2.07]$ & $<.001$ & 0 & .627 & .079 & & $\longmapsto$ \\
\hline \multicolumn{7}{|l|}{ Types pf adenoma } & & \\
\hline Any adenoma & 17 & $1.42[1.34,1.51]$ & $<.001$ & 34.3 & .063 & Ref & & $=$ \\
\hline Nonadvanced & 8 & $1.36[1.26,1.47]$ & $<.001$ & 25.4 & .183 & .374 & & $=$ \\
\hline Advanced & 8 & $1.52[1.32,1.73]$ & $<.001$ & 15.4 & .056 & .401 & & $\mapsto-1$ \\
\hline \multirow[t]{3}{*}{ Adv. vs Non-adv. } & 8 & $1.12[0.99,1.26]$ & .073 & 0 & .258 & $<.001$ & & $=-1$ \\
\hline & & & & & & & г & 1 \\
\hline & & & & & & & 0.5 & 1 \\
\hline
\end{tabular}

Fig. 3 Subgroup analysis - association between BMI and colorectal adenoma according to study design, gender, ethnicity, and types of adenoma $(\mathrm{BMI} \geq 25$ vs. $<25)$ 
SOR was $1.41(95 \%$ CI $1.34,1.49)$ which was statistically similar to the SOR computed from all studies.

\section{Discussion}

This systematic review and meta-analysis based on high quality studies reported increased risks of any CRA and non-advanced adenomas in the overweight and obese populations by a magnitude of $33-44 \%$ - risk estimates that are significantly higher than those reported previously. BMI was found to be a significant factor associated with detection of CRA in terms of its magnitude, and hence should be considered as an important factor in risk algorithms predicting the risk of CRA. The strength of association between BMI and CRA was higher in female subjects and individuals of western or Asian ethnicities, but was insignificant in subjects of African descent.

This meta-analysis is distinct from previous systematic reviews by restricting analysis to the most updated studies retrieved from a broad search strategy that included the most comprehensive data. This enables more robust evaluations on the association between BMI and CRA, allowing a more precise magnitude to be determined. Several limitations should, nevertheless, be addressed. Firstly, the assessment of BMI and CRA might not be universally standardized among different studies, and it is well recognized that there is a higher likelihood for obese patients, or subjects with different characteristics, to present with poorer bowel preparation at colonoscopy procedures [58, 59]. Therefore, the summary odds ratios identified in the present study might have been underestimated. Second, the calendar years where CRA were detected are different across studies, where colonoscopists with different levels of experience and expertise were involved. The adenoma detection rate might increase with time due to higher prevalence with rapid urbanization and more affluent lifestyles. Also, there have been very few prospective cohort studies that followed-up screening subjects and examine the direct influence of obesity on CRA development [57]. Furthermore, the estimation of dose-response association requires at least three non-reference dose levels [60]. As most original studies included in this meta-analysis only used two nonreference dose levels $(\mathrm{BMI} 25-30, \mathrm{BMI}>30$, reference: BMI < 25), dose-response meta-analysis could not be performed. From one cohort study (Sedjo et al. [57]), the association between CRA and obesity vs. overweight (adjusted OR 2.16, 95\% CI 1.13-4.14 vs. OR 1.54, 95\% CI 0.81-2.91) suggested a trend towards dose-response relationship, although statistical analysis did not confirm such relationship. The cross-sectional nature of most studies included in this meta-analysis might obscure a potential dose-response association. In addition, multivariate meta-regression analysis could not be performed since we need an appropriately large ratio of studies to covariates [61]. In this meta-analysis it is not feasible due to multiple covariates and the small number of studies. Lastly, as the majority of studies included in this metaanalysis are cross-sectional or case-control studies, one could not infer a cause-and-effect relationship between BMI and CRA.

The exact mechanisms of colorectal carcinogenesis induced by obesity are still not entirely clear. Our study findings reported a significant association between BMI and CRA, but when the outcome measure is development of non-advanced CRA to advanced CRA, the association becomes insignificant. This implies that obesity could exert, to a larger extent, its influence on risk of adenoma, but less so on adenoma progression. There has been a postulation that genetic alteration like the common single-nucleotide polymorphism variants around the melanocortin 4 receptor gene could be associated with the cooccurrence of obesity and CRA [32, 62]. Alternatively, it has been hypothesized that insulin resistance and subsequent hyperinsulinemia induced by obesity may lead to direct mitogenic and antiapoptotic signaling by insulin or insulin-like growth factor axis [63, 64]. Furthermore, obesity has been regarded as a condition of chronic lowgrade inflammation with elevation of pro-inflammatory cytokines, including tumor necrosis factor and interleukin-6. These inflammatory mediators have direct tumorigenic effects on the gastrointestinal tract [63, 64]. From a recent meta-analysis, leptin and adiponectin have also been implicated in the pathogenesis of CRA in obese patients [65]. In addition, there are metabolic, lipidomic and transcriptomic differences between visceral adipose tissue (VAT) and subcutaneous adipose tissue (SAT) compartments in colorectal carcinogenesis [66], which have not been differentiated in this study. There is emerging evidence demonstrating that the relationship between obesity and cancer is mediated by VAT rather than SAT. Several studies have identified a unique role of VAT in the risk and progression of CRC. It has been postulated that VAT alters metabolic activity and induces chronic systemic inflammation that promotes a pro-oncogenic environment [67]. Future studies may explore the magnitude of association between VAT and CRA.

We found that a 5-unit increase of BMI conferred an up to $44 \%$ increased risk for CRA. This additional risk is significantly higher than that estimated by previous metaanalyses [9, 32]. The increased risk estimated by Okabayashi et al and Ben et al in 2012 was 24 and $19 \%$, respectively. The difference could be explained by different inclusion criteria of original studies in these meta- 
analyses. In their evaluations, studies that included selfreported BMI and questionnaire-measured CRA were also included in their systematic review. Studies showed that BMI based on self-reports were more frequently underreported, where data from measurement devices usually revealed higher proportions of overweight and obesity $[68,69]$. Hence, the true association between BMI and CRA might be biased towards lower risk. In addition, except on cohort study, this meta-analysis mainly included case-control and cross-sectional studies. Risk estimates are therefore higher in retrospective studies as compared to previous meta-analyses, which also included prospective studies.

Our study also found that the association between BMI and CRA was significantly higher in women than men, in the context of higher prevalence of CRC in men when compared with women. It has been suggested that this gender difference might be due to the role of endogenous and exogenous sex hormones on the adenocarcinoma sequence [32]. It is well recognized that pre-menopausal women had a stronger susceptibility to CRA development due to endogenous estrogen secretion, where activation of estrogen receptor- $\alpha$ leads to increase in gene transcription and cancer proliferation [70]. As for the differences in the association between BMI and CRA, ethnicity of individuals was found to be a significant effect modifier. In particular, the association between BMI and CRA was found to be absent in subjects of African descent. The difference in prevalence of overweight and obesity in individuals according to ethnicity might affect the comparability among studies that included screening participants of different ethnic groups. From existing literature, the magnitude of this association has not been adequately examined, and the exact reasons of this observation will need to be explored in future studies.

These study findings showed that being overweight (BMI 25-30) is associated with similar risk for CRA when compared with obesity (BMI $\geq 30$ ), and hence bring forth an alert to physicians and public health practitioners on early intervention for overweight patients in order to reduce future risk of CRA. In addition, our data showed that risk algorithms for CRA would need to take gender and ethnicities into account for more accurate risk prediction, and these findings could be used for devising such risk-stratification scores. Future studies should examine the mechanistic aspects of the differential effects of these variables on CRA development. As there is a scarcity of prospective studies on the impact of BMI on progression of CRA to advanced CRA, additional longitudinal cohort evaluations should be performed with strategies that address confounding and selection biases.

\section{Compliance with ethical standards}

Conflict of interest None declared from all authors.

Open Access This article is distributed under the terms of the Creative Commons Attribution 4.0 International License (http://creative commons.org/licenses/by/4.0/), which permits unrestricted use, distribution, and reproduction in any medium, provided you give appropriate credit to the original author(s) and the source, provide a link to the Creative Commons license, and indicate if changes were made.

\section{References}

1. Ferlay J, Soerjomataram I, Ervik M, et al. GLOBOCAN 2012 v1.0, cancer incidence and mortality worldwide. IARC cancer base no 11. Lyon: International Agency for Research on Cancer; 2013.

2. Arnold M, Sierra MS, Laversanne M, et al. Global patterns and trends in colorectal cancer incidence and mortality. Gut. 2017;66:683-91.

3. Center MM, Jemal A, Smith RA, et al. Worldwide variations in colorectal cancer. CA Cancer J Clin. 2009;59:366-78.

4. Sung JJY, Lau JYW, Goh KL, for the on Asia Pacific Working Group on Colorectal Cancer, et al. Increasing incidence of colorectal cancer in Asia: implications for screening. Lancet Oncol. 2005;6:871-6.

5. World Cancer Research Fund/American Institute for Cancer Research. Continuous update project report. Food, nutrition, physical activity, and the prevention of colorectal cancer. 2011. http://wcrf.org/sites/default/files/Colorectal-Cancer-2011-Report. pdf. Accessed 27 Sept 2017.

6. Wong MC, Wong SH, Ng SC, et al. Targeted screening for colorectal cancer in high-risk individuals. Best Pract Res Clin Gastroenterol. 2015;29:941-51.

7. Wong MC, Lam TY, Tsoi KK, et al. Predictors of advanced colorectal neoplasia for colorectal cancer screening. Am J Prev Med. 2014;46:433-9.

8. World Health Organization. Obesity and overweight fact. http:// www.who.int/mediacentre/factsheets/fs311/en/. Accessed 29 Mar 2017.

9. Wong MC, Lam TY, Tsoi KK, et al. A validated tool to predict colorectal neoplasia and inform screening choice for asymptomatic subjects. Gut. 2014;63:1130-6.

10. Sung JJ, Wong MC, Lam TY, et al. A modified colorectal screening score for prediction of advanced neoplasia: a prospective study of 5,744 subjects. J Gastroenterol Hepatol. 2017. https://doi.org/10.1111/jgh.13835.

11. Neugut AI, Lee WC, Garbowski GC, et al. Obesity and colorectal adenomatous polyps. J Natl Cancer Inst. 1991;83:359-61.

12. Olsen J, Kronborg O, Lynggaard J, et al. Dietary risk factors for cancer and adenomas of the large intestine: a case-control study within a screening trial in Denmark. Eur $\mathrm{J}$ Cancer. 1994;30A:53-60.

13. Giovannucci E, Colditz GA, Stampfer MJ, et al. Physical activity, obesity, and risk of colorectal adenoma in women (United States). Cancer Causes Control. 1996;7:253-63.

14. Bird CL, Frankl HD, Lee ER, et al. Obesity, weight gain, large weight changes, and adenomatous polyps of the left colon and rectum. Am J Epidemiol. 1998;147:670-80.

15. Schoen RE, Weissfeld JL, Kuller LH, et al. Insulin-like growth factor-I and insulin are associated with the presence and 
advancement of adenomatous polyps. Gastroenterology. 2005;129:464-75.

16. Chung YW, Han DS, Park YK, et al. Association of obesity, serum glucose and lipids with the risk of advanced colorectal adenoma and cancer: a case-control study in Korea. Dig Liver Dis. 2006;38:668-72.

17. Larsen IK, Grotmol T, Almendingen K, et al. Lifestyle as a predictor for colonic neoplasia in asymptomatic individuals. BMC Gastroenterol. 2006;6:5.

18. Lee GE, Park HS, Yun KE, et al. Association between BMI and metabolic syndrome and adenomatous colonic polyps in Korean men. Obesity (Silver Spring). 2008;16:1434-9.

19. Wise LA, Rosenberg L, Palmer JR, et al. Anthropometric risk factors for colorectal polyps in African-American women. Obesity (Silver Spring). 2008;16:859-68.

20. Hermann S, Rohrmann S, Linseisen J. Lifestyle factors, obesity and the risk of colorectal adenomas in EPIC-Heidelberg. Cancer Causes Control. 2009;20:1397-408.

21. Huang L, Wang X, Gong W, et al. The comparison of the clinical manifestations and risk factors of colorectal cancer and adenomas: results from a colonoscopy-based study in southern Chinese. Int J Colorectal Dis. 2010;25:1343-51.

22. Le Marchand L, Wang H, Rinaldi S, et al. Associations of plasma C-peptide and IGFBP-1 levels with risk of colorectal adenoma in a multiethnic population. Cancer Epidemiol Biomark Prev. 2010;19:1471-7.

23. Morois S, Mesrine S, Josset M, et al. Anthropometric factors in adulthood and risk of colorectal adenomas: the French E3N-EPIC prospective cohort. Am J Epidemiol. 2010;172:1166-80.

24. Nam SY, Kim BC, Han KS, et al. Abdominal visceral adipose tissue predicts risk of colorectal adenoma in both sexes. Clin Gastroenterol Hepatol. 2010;8:443-50.

25. Tsilidis KK, Brancati FL, Pollak MN, et al. Metabolic syndrome components and colorectal adenoma in the CLUE II cohort. Cancer Causes Control. 2010;21:1-10.

26. Wernli KJ, Newcomb PA, Wang Y, et al. Body size, IGF and growth hormone polymorphisms, and colorectal adenomas and hyperplastic polyps. Growth Horm IGF Res. 2010;20:305-9.

27. Yamamoto S, Nakagawa T, Matsushita Y, et al. Visceral fat area and markers of insulin resistance in relation to colorectal neoplasia. Diabetes Care. 2010;33:184-9.

28. Anderson JC, Messina CR, Dakhllalah F, et al. Body mass index: a marker for significant colorectal neoplasia in a screening population. J Clin Gastroenterol. 2007;41:285-90.

29. Morimoto LM, Newcomb PA, Ulrich CM, et al. Risk factors for hyperplastic and adenomatous polyps: evidence for malignant potential? Cancer Epidemiol Biomark Prev. 2002;11:1012-8.

30. Otake S, Takeda H, Suzuki Y, et al. Association of visceral fat accumulation and plasma adiponectin with colorectal adenoma: evidence for participation of insulin resistance. Clin Cancer Res. 2005;11:3642-6.

31. Chia VM, Newcomb PA, Lampe JW, et al. Leptin concentrations, leptin receptor polymorphisms, and colorectal adenoma risk. Cancer Epidemiol Biomark Prev. 2007;16:2697-703.

32. Okabayashi K, Ashrafian H, Hasegawa H, et al. Body mass index category as a risk factor for colorectal adenomas: a systematic review and meta-analysis. Am J Gastroenterol. 2012;107:1175-85.

33. Gosse M. How accurate is self-reported BMI? Nutr Bull. 2014;39:105-14.

34. Ben Q, An W, Jiang Y, et al. Body mass index increases risk for colorectal adenomas based on meta-analysis. Gastroenterology. 2012;142:762-72.

35. Wong MC, Ching JY, Chiu HM, et al. Risk of colorectal neoplasia in individuals with self-reported family history: a prospective colonoscopy study from 16 Asia-Pacific regions. Am J Gastroenterol. 2016;111:1621-9.

36. Stang A. Critical evaluation of the Newcastle-Ottawa scale for the assessment of the quality of nonrandomized studies in metaanalyses. Eur J Epidemiol. 2010;25:603-5.

37. Wells G, Shea B, O'Connell D et al. The Newcastle-Ottawa Scale (NOS) for assessing the quality of nonrandomised studies in meta-analyses. 2014. http://www.ohri.ca/programs/clinical_ epidemiology/oxford.asp. Accessed 29 Mar 2017.

38. Higgins JP, Thompson SG, Deeks JJ, et al. Measuring inconsistency in meta-analyses. BMJ. 2003;327:557-60.

39. Egger M, Davey Smith G, Schneider M, et al. Bias in metaanalysis detected by a simple, graphical test. BMJ. 1997;315: 629-34.

40. Begg CB, Mazumdar M. Operating characteristics of a rank correlation test for publication bias. Biometrics. 1994;50: $1088-101$.

41. Viechtbauer W. Conducting meta-analyses in $\mathrm{R}$ with the metafor package. J Stat Softw. 2010;36:1-48.

42. Liberati A, Altman DG, Tetzlaff J, et al. The PRISMA statement for reporting systematic reviews and meta-analyses of studies that evaluate health care interventions: explanation and elaboration. Ann Intern Med. 2009;151:W65-94.

43. Guilera M, Connelly-Frost A, Keku TO, et al. Does physical activity modify the association between body mass index and colorectal adenomas? Nutr Cancer. 2005;51:140-5.

44. Kim S, Shim K, Jung S, et al. An association between obesity and the prevalence of colonic adenoma according to age and gender. J Gastroenterol. 2007;42:616-23.

45. Kim K, Moon $\mathrm{HJ}$, Choi $\mathrm{CH}$, et al. The frequency and risk factors of colorectal adenoma in health-check-up subjects in South Korea: relationship to abdominal obesity and age. Gut Liver. 2010;4:36-42.

46. Stein B, Anderson JC, Rajapakse R, et al. Body mass index as a predictor of colorectal neoplasia in ethnically diverse screening population. Dig Dis Sci. 2010;55:2945-52.

47. Kim YJ, Lee KM, Chung WC, et al. Association between measures of obesity and colorectal adenoma. Chin Med J. 2011;124:3711-5.

48. Kim MC, Kim CS, Chung TH, et al. MONW phenotype is associated with advanced colorectal adenoma in Korean men. Obesity. 2012;20:1876-81.

49. Yun KE, Chang Y, Jung HS, et al. Impact of body mass index on the risk of colorectal adenoma in a metabolically healthy population. Cancer Res. 2013;73:4020-7.

50. Lee JY, Kwak SM, Myung SK, et al. Obesity and colorectal adenomatous polyps: a cross-sectional study in Korean adults. Obesity. 2014;22:518-25.

51. Wang FW, Hsu PI, Chuang H.Y. et al. Prevalence and risk factors of asymptomatic colorectal polyps in Taiwan. Gastroenterol Res Pract. 2014;2014 (2014):8. https://doi.org/10.1155/2014/985205

52. Murphy CC, Martin CF, Sandler RS. Racial differences in obesity measures and risk of colorectal adenomas in a large screening population. Nutr Cancer. 2015;67:98-104.

53. Choe EK, Kim D, Kim HJ, et al. Association of visceral obesity and early colorectal neoplasia. World J Gastroenterol. 2013;19:8349-56

54. Czwornog J, Austin GL. Body mass index, age, and gender affect prep quality, sedation use, and procedure time during screening colonoscopy. Dig Dis Sci. 2013;58:3127-33.

55. Lipka S, Zheng XE, HurtadoCordovi J, et al. Obesity, metabolic factors, and colorectal adenomas: a retrospective study in a racially diverse new york state hospital. J Gastrointest Cancer. 2013;44:270-6.

56. Kim DH, Cha JM, Shin HP, et al. Development and validation of a risk stratification-based screening model for predicting 
colorectal advanced neoplasia in Korea. J Clin Gastroenterol. 2015;49:41-9.

57. Sedjo RL, Byers T, Levin TR, et al. Change in body size and the risk of colorectal adenomas. Cancer Epidemiol Biomark Prev. 2007;16:526-31.

58. Borg BB, Gupta NK, Zuckerman GR, et al. Impact of obesity on bowel preparation for colonoscopy. Clin Gastroenterol Hepatol. 2009;7:670-5.

59. Wong MC, Ching JY, Chan VC, et al. Determinants of bowel preparation quality and its association with adenoma detection: a prospective colonoscopy study. Medicine (Baltimore). 2016;95:e2251.

60. Crippa A, Orsini N. Dose-response meta-analysis of differences in means. BMC Med Res Methodol. 2016;16:91.

61. Borenstein M, Hedges LV, Higgins JPT and Rothstein HR. Introduction to meta-analysis. Chichester: Wiley, 2009. ISBN: 978-0-470-05724-7.

62. Tenesa A, Campbell H, Theodoratou E, et al. Common genetic variants at the MC4R locus are associated with obesity, but not with dietary energy intake or colorectal cancer in the Scottish population. Int J Obes. 2009;33:284-8.

63. Renehan AG, Frystyk J, Flyvbjerg A. Obesity and cancer risk: the role of the insulin-IGF axis. Trends Endocrinol Metab. 2006;17:328-36.
64. Clayton PE, Banerjee I, Murray PG, et al. Growth hormone, the insulin-like growth factor axis, insulin and cancer risk. Nat Rev Endocrinol. 2011;7:11-24.

65. An W, Bai Y, Deng SX, et al. Adiponectin levels in patients with colorectal cancer and adenoma: a meta-analysis. Eur J Cancer Prev. 2012;21:126-33.

66. Liesenfeld DB, Grapov D, Fahrmann JF, et al. Metabolomics and transcriptomics identify pathway differences between visceral and subcutaneous adipose tissue in colorectal cancer patients: the ColoCare study. Am J Clin Nutr. 2015;102:433-43.

67. Vongsuvanh R, George J, Qiao L, van der Poorten D. Visceral adiposity in gastrointestinal and hepatic carcinogenesis. Cancer Lett. 2013;330:1-10.

68. Scribani M, Shelton J, Chapel D, et al. Comparison of bias resulting from two methods of self-reporting height and weight: a validation study. JRSM Open. 2014;5(6):2042533313514048.

69. John U, Hanke M, Grothues J, et al. Validity of overweight and obesity in a nation based on self-report versus measurement device data. Eur J Clin Nutr. 2006;60:372-7.

70. Folkerd EJ, Dowsett M. Influence of sex hormones on cancer progression. J Clin Oncol. 2010;28:4038-44.

\section{Affiliations}

\section{Martin Chi-sang Wong ${ }^{1,2} \cdot$ Chun-hei Chan ${ }^{2} \cdot$ Wilson Cheung ${ }^{2} \cdot$ Din-hei Fung $^{2} \cdot$ Miaoyin Liang $^{2} \cdot$ Jason Li-wen Huang ${ }^{2} \cdot$ Yan-hong Wang $^{3} \cdot$ Johnny Yu Jiang ${ }^{4} \cdot$ Chun-pong $\mathrm{Yu}^{5} \cdot$ Harry Haoxiang Wang ${ }^{6}$. Justin Che-yuen $\mathrm{Wu}^{1,7,8} \cdot$ Francis Ka-leung Chan ${ }^{1,7,8} \cdot$ Joseph Jao-yiu Sung ${ }^{1,7,8}$}

1 Institute of Digestive Disease, Faculty of Medicine, Chinese University of Hong Kong, Shatin, China

2 School of Public Health and Primary Care, Faculty of Medicine, Chinese University of Hong Kong, Shatin, China

3 School of Basic Medicine, Peking Union Medical College and Institute of Basic Medical Sciences, Chinese Academy of Medical Sciences, Beijing 100050, China

4 Peking Union School of Public Health, Chinese Academy of Medical Sciences and Peking Union Medical College, Beijing 100050, China
5 Li Ping Medical Library, Chinese University of Hong Kong, Shatin, HKSAR, China

6 School of Public Health, Sun Yat-sen University, Guangzhou 510080, Guangdong, China

7 Department of Medicine and Therapeutics, Faculty of Medicine, Chinese University of Hong Kong, Shatin, China

8 State Key Laboratory of Digestive Disease, Faculty of Medicine, Chinese University of Hong Kong, Shatin, China 